\title{
Contribution of Simulation in the Development of the Competences of Future Radiology Technicians in the Framework of the Management of the Risks Associated with the Medical Radiology Techniques
}

\author{
https://doi.org/10.3991/ijoe.v17i08.23779 \\ Asma Fadi ${ }^{1}{ }^{凶}$ ), Khadija Legrouri ${ }^{1}$, Jabran Daaif ${ }^{1}$, Said Benmokhtar ${ }^{1}$, \\ Said Belaaouad $^{1}$, Fatima Zahra Ramdani ${ }^{2}$, El Hassane Khouya ${ }^{3}$ \\ ${ }^{1}$ Hassan II University of Casablanca, Morocco. \\ ${ }^{2}$ The Higher Institute of Nursing and Health Techniques of Agadir, Morocco \\ ${ }^{3}$ Regional Center for Education and Training - Souss Massa. Morocco \\ asmafadi2017@gmail.com
}

\begin{abstract}
The objectives of this study are to raise awareness among future radiology technicians regarding risk management in medical imaging and to measure their satisfaction with their participation in simulation sessions focused on the management of emergencies in radiology. A prospective research-action study was conducted at the simulation center of the Institut Supérieur des Professions Infirmières et Techniques de Santé of Agadir, with radiology technician students enrolled in the 5th semester. A simulation workshop applied to the theme "Risk management in radiology related to an immediate hypersensitivity reaction to iodinated contrast products (ICP) used in CT" was organized over three days. Sixteen radiology students participated in the three simulation scenarios. The evaluation of the technical skills of the participants in this simulation workshop revealed an insufficient mastery of these emergencies. Indeed, during the first simulation session concerning the management of an immediate hypersensitivity reaction to iodinated contrast products in second-grade $\mathrm{CT}$, the average score for the session was $06.39 / 20$ (3.25-9). In addition, the mean score for the second simulation session applied to cardiac arrest management was 06.03/20 (2.25- 8.75). Regarding the error room scenario, (50\%) of the participants were satisfied with the relevance of the errors implemented in this simulation session, (37.5\%) very satisfied, and (75\%) judged the simulation as an effective approach to risk management. Teaching by simulation applied to the management of medical imaging emergencies proves to be a relevant pedagogical method for building a base of skills for future radiology professionals.
\end{abstract}

Keywords—simulation, training, risk management, medical radiology 


\section{$1 \quad$ Introduction}

\subsection{Management of risks associated with care}

The health care system is a complex environment with a high risk of error, the consequences of which can be life-threatening or even fatal for the patient. [1]

Indeed, there is a growing awareness and commitment to patient safety worldwide since the late 1990s, following the emergence of the Academy of Medicine's U.S. study "To Err is Human: Building a Safer Health System" [2, 3]. The results of this study sounded the alarm by revealing that in this country, the estimated number of deaths due to adverse events associated with care (AEHC) in hospitals amounts to 98,000 each year when they could have been avoided [4]. The authors compare them with the number of deaths related to road accidents $(43,458)$, breast cancer $(42,297)$, and AIDS (16,516), thus constituting the 8th leading cause of death [4]. The UK Department of Health, in its 2000 report "An organization with a memory," estimated that adverse events occur in about $10 \%$ of hospitalized patients, representing about

850,000 adverse events each year $[5,6]$.

The 1991 Harvard Medical Practice study found that 4\% of patients experience some type of harm in the hospital; $70 \%$ of adverse events lead to brief disability, but $14 \%$ result in death [6].

Similarly, studies in several countries have shown a rate of adverse events ranging from $3.5 \%$ to $16.6 \% 2$ among hospitalized patients. On average, one in ten hospitalized patients suffers avoidable harm that can result in severe disability or even death $[5,1]$.

An Australian study, Quality in Australian Health Care Study (QAHCS), published in 1995, found an adverse event rate of $16.6 \%$ among hospitalized subjects [6].

The Hospitals for Europe Working Group on Quality of Care in Hospitals estimates that in 2000, one in ten patients in European hospitals suffered avoidable harm and adverse events related to the care they received [6].

In France, the two ENEIS surveys in 2004 and 2009 showed that a serious adverse event (SAE) occurred every 5 days in a 30 -bed unit, and this was fairly stable. $40 \%$ of these events are avoidable $[4,7]$.

Moreover, a survey conducted by the Institute for Research and Documentation in Health Economics (IRDES) in 2011 estimates the cost of SAEs in France at 700 million euros in 2007 alone [8].

Beyond the resulting damage, adverse events also have important economic consequences. Several studies estimate the cost to some countries of additional hospitalizations, court awards, hospital-acquired infections, lost income, disability, and medical expenses to be between $\$ 6$ billion and $\$ 29$ billion per year $[1,5]$.

In the United Kingdom of Great Britain and Northern Ireland, prolonged hospital stays alone cost some $£ 2$ billion per year, and compensation payments cost the National Health Service some $£ 400$ million per year, not including the potential compensation claims - estimated at $£ 2.4$ billion - that may arise from pending and expected claims, while hospital-acquired infections - $15 \%$ of which may be preventable - have an estimated annual cost of nearly $£ 1$ billion [6]. 
The total cost of preventable adverse medical events in the United States of America, including lost income, disability-related expenses, and medical costs, is estimated to be between US \$17 billion and US \$29 billion each year. Added to these costs is the loss of public and provider trust and satisfaction [6].

A 2017 OECD report estimates that $15 \%$ of healthcare spending and activity at healthcare facilities is related to the treatment and management of HAIs $(4,9)$.

As a result, the quest to improve the management of risks associated with care and to engender a culture of safety in care is a priority for most health systems.

In view of this situation, patient safety and risk management are a priority for most healthcare systems and a public health issue [1].

In the field of medical radiology, the safety of patients and professionals is a fundamental issue in imaging procedures. It is therefore essential to ensure that safety and risk prevention rules are respected.

The occurrence of such events is rarely linked to a lack of knowledge on the part of professionals. It is systematically the result of a combination of various causes, linked to the direct quality of the care, but also to general organizational defects in the material and human environment in which the care was provided. Numerous studies have highlighted the frequency, severity and often avoidable nature of adverse events associated with care $[3,4,10]$.

Several examples of radiological emergencies illustrate this picture: risks related to the use of X-rays (radiation protection), the use of contrast products (hypersensitivity), magnetic fields and radiofrequency in MRI (magnetoprotection), interventional radiology or the control of the patient's identity (identity surveillance).

In addition, due to the lack of experience for young and future professionals or the rarity of these events in the daily practice of radiology technicians, their skills and ability to cope with and manage these urgent situations are insufficiently mastered or diminish with time and thus the risk of error increases.

In this perspective, teaching through simulation compensates for this lack of exposure to these rare and critical care situations and proves to be an effective and essential pedagogical method for the acquisition of experience without risk to the patient and the construction of a base of competences for radiology professionals and learners enabling them to better manage complex, unpredictable and rare critical situations in real professional life $[11,12,13,14]$.

\subsection{Simulation in the teaching of medical radiology techniques}

Simulation in healthcare is a relevant, effective and essential teaching method and approach that has experienced a remarkable boom in recent years in the initial and continuing education of healthcare professionals.

Simulation, on the other hand, can be adopted in a formal way not only in the field of health but also in science and technology in general. Indeed, in physics or chemistry there are virtual environment laboratories allowing the study and description of different experiments and manipulations, such as in crystallography $[49,50]$.

In addition, virtual simulation environments can be adapted according to the study context; computers or smartphones which represent a motivating and engaging space 
in a pedagogical and didactic framework, by adopting the principle of mobile-learning and social-learning [53].

In its report on good practice in healthcare simulation, the Haute Autorité de Santé defined healthcare simulation as "the use of equipment (such as a mannequin or procedural simulator), virtual reality or a standardized patient, to reproduce healthcare situations or environments, to teach diagnostic and therapeutic procedures and to allow rehearsal of processes, clinical situations or decision making by a healthcare professional or team of professionals" [15,16,17].

For Gaba, simulation is a technique, but not a technology, for replacing or amplifying a real experience with a guided experience that evokes or replicates a substantial aspect of the real world in a fully interactive way [18].

For Pascal Béguin and Annie Weill Fassina:

"It is a method of teaching skills and abilities used in tasks for which direct instruction is impossible for ethical, economic or technical reasons. The objective is to allow the operator to learn to reproduce the expected behaviors as realistically and faithfully as possible [19].

For Jacques Leplat "The notion of simulation covers most often the case where a support object, the simulator, is used, specified by a goal linked very directly to the work; but there are other categories of simulation in which the role of the support object is no longer played by a material device but by a human being or group or by a symbolic or virtual situation" [20].

In the field of medical radiology, several categories of simulation are advocated in simulation-based education in the training curriculum of future radiology technicians.

The use of virtual reality simulation is the preferred method of teaching diagnostic medical imaging, particularly mammography [21,22]. This mode of teaching has been shown to have a favorable effect on the acquisition of radiological skills such as positioning [23,24,25]. It provides secure access to graphic resources, radiological images and text. It also offers the possibility of exposing students to role-playing situations, encouraging them to annotate radiological images and to provide a reflective view of these images in the form of exchanges between peers or with their teachers [23].

Similarly, anatomical "phantoms" of body parts or whole human bodies are suitable for comprehensive evaluation of patient positioning during X-ray or CT scanning [26]. They allow the students to be tested on different parameters related to the radiological technique such as contrast or optical density.

Research has studied the contribution of the "E-Assessment" on the appreciation of the personality and the value system within professional organizations. Indeed, the platforms dedicated to evaluation cover several families: behavioral tests or personality tests, aptitude tests and value questionnaires [56].

Finally, the Objective Structured Clinical Examinations (OSCE), a standardized tool for evaluating declarative and procedural knowledge (Miller's Pyramid shows) in a simulated situation, have been developed as part of the initial training of radiology students. These are activities that require handling skills, patient positioning, equipment handling, technical adjustments as well as safety and prevention. 
Paper- Contribution of Simulation in the Development of the Competences of Future Radiology...

Other ethical and relational aspects can also be evaluated in the context of OSCEs, such as the search for informed consent, informing the patient about the care, and team communication [27].

However, it has been noted that in the field of medical radiology, the preferred simulation methods are not adapted to the acquisition of clinical skills in the management of risk situations, nor of non-technical skills such as communication and teamwork [27]. Indeed, confrontations with others generally represent necessary moments that collaborators look for justifications to consolidate in the way of interaction [51].

In view of this situation, the development of other categories of simulation such as mixed or hybrid simulation which associates an actor or a standardized patient proves to be important for the development of a set of technical and non-technical skills allowing to better prepare radiology students for clinical practice, to confront critical, complex situations with a high risk of error and rare in professional life, and to develop in them the notion of teamwork, a major factor in the safety of care $[28,29,30]$.

\subsection{Objectives}

The objectives of this study were to raise the awareness of future radiology technicians regarding risk management in medical imaging and to measure their satisfaction with their participation in these simulation sessions focused on the management of emergency situations in radiology.

\subsection{Materials and methods}

This is a prospective monocentric study of the action research type that was conducted at the simulation center of the Higher Institute of Nursing Professions and Health Techniques in Agadir, Morocco.

\subsection{Sampling and recruitment}

All student technicians enrolled in the 5th semester of the radiology option, 16 students, were invited to participate in this study. Participation in this study was optional and did not affect the validation of their module.

\subsection{Simulation workshop in radiology risk management}

The simulation workshop was on the topic of "Risk Management in Radiology" and was conducted over a three-day period. Three simulation sessions were offered to the student radiology technicians during the study. In this study, we adopted the a priori approach where simulation is used in a preventive approach [4]. The workshop included different scenarios that cover some emergency situations that future radiology technicians may face during their practice in a radiology department. The scenarios were designed in collaboration with a multidisciplinary team with expertise in clinical teaching, as well as in school and university guidance. Indeed, more importance had 
to be given to the orientation of the participants, knowing the importance of the cooperation of teachers in the orientation process [58].

In this study, the development and implementation of this simulation workshop is based on guidelines derived from the Standards of Best Practice: Simulation published by the International Nursing Association for Clinical Simulation and Learning [31], a global leader in transforming practice to improve patient safety through excellence in healthcare simulation. The seven standards were produced by committees of INACSL members (Society for Simulation in Healthcare (SSIH), National League for Nursing (NLN), California Simulation Alliance (CSA), Haute autorité en santé (HAS) [15].

Similarly, this session was organized while respecting the three distinct stages of a simulation session: the briefing, which allows the trainer to specify the framework of the session and its precise objectives, the unfolding of the scenario and finally the debriefing, which is a crucial moment of learning and reflection on the simulation session $[15,21,32]$.

During this simulation workshop "Risk management in radiology", and according to the educational objectives assigned to this workshop, we combined several types of simulation. Thus, the research team opted for hybrid simulation, which corresponds to the use of several simulation techniques by combining the use of a procedural simulator/mannequin with the use of a standardized patient who plays the role of the patient to increase the realism of the situation $[3,21]$.

Also, the research team called upon actors (resuscitation physician, radiology physician, radiology technician) who were trained and prepared to carry out the simulation sessions. The evaluations of the simulation sessions were carried out by the same evaluators trained in simulation.

\subsection{The choice of scenarios and justification for the choice of contrast media and risk management in $\mathrm{CT}$}

The scenarios were chosen in view of the rarity of these high-risk clinical situations and according to the constraints inherent to the training site and the real care situation, which generates an obligation of efficiency and rapidity of diagnosis and therapeutic management without leaving the possibility for the radiology trainee to act by making errors.

In this simulation workshop, we discussed the risks associated with an immediate hypersensitivity reaction that can quickly put the patient's life at risk. These reactions are based on immunological mechanisms. They are individualized into delayed cellmediated reactions and immediate reactions triggered by specific immunoglobulins of type E (IgE) [33].

The European Academy of Allergology and Clinical Immunology (EAACI) has defined the classification of hypersensitivity reactions into all allergic-like reactions. A distinction is made between non-allergic hypersensitivity reactions and allergic hypersensitivity reactions (Figure 1) [33].

Indeed, medical imaging is based on the study of tissue contrasts related to the physical processes used and the physicochemical nature of the tissues. On an X-ray image, structures with high natural contrast can be studied spontaneously (bone tissue, 
aerial structures). Structures with low density difference (soft tissue) cannot be studied spontaneously. To compensate for this deficit, the administration of lower density (air) or higher density (barium) contrast media is necessary. The increase in contrast is due to the presence of atoms with high atomic numbers $(Z=53$ for iodine, 54 for barium) which increase the absorption of X-ray photons $[34,35,36]$.

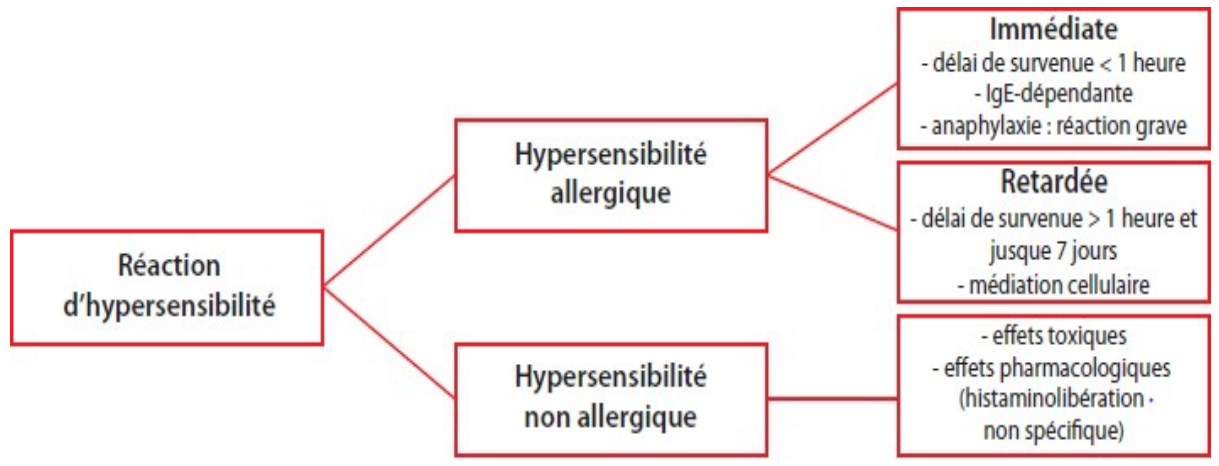

Fig. 1. Classification of hypersensitivity reactions according to the European Academy of Allergy and Clinical Immunology (EAACI).

Contrast media are chemicals belonging to the class of drugs. They are injectable or ingestible substances that improve the visualization of the organs being explored [36].

There are several categories of contrast agents. The most commonly used at present are iodinated contrast products (ICP) used in $\mathrm{CT}$ and gadoline contrast products (GCP) used in MRI. Recently, a gaseous contrast medium has been used in ultrasound. The oldest of these contrast products are those used for exploration of the digestive tract based on barium sulfate [36].

The injection of a contrast product is a frequent procedure during routine examinations such as CT and MRI. Accidents are rare and even exceptional for the most serious ones. The risk factors are known, but for certain reactions, they are unpredictable.

The Ring and Messmer classification according to the European Academy of Allergology and Clinical Immunology (EAACI) allows the clinical signs of immediate hypersensitivity reactions to be stratified into four grades of increasing clinical severity.

The first two grades (I and II) are generally not life-threatening, unlike the last two (III and IV). This classification should be known because it also helps to guide therapeutic management $[37,38]$ (See Table 1).

Thus, the injection stage is a high-risk situation for the radiology technician and remains a critical moment for the teams. This awareness of risk-taking, which is so prevalent in medical imaging, should encourage management. Managing means knowing, preventing and organizing [39]. 
Paper- Contribution of Simulation in the Development of the Competences of Future Radiology...

Table 1. Ring and Messmer classification of immediate hypersensitivity reactions according to their severity.

\begin{tabular}{|l|l|}
\hline \multicolumn{2}{|c|}{ Classification of Ring and Messmer } \\
\hline Grades & \multicolumn{1}{|c|}{ Symptoms } \\
\hline II & $\begin{array}{l}\text { Moderate multivisceral involvement Mucocutaneous signs } \pm \text { arterial hypotension } \pm \text { tachycardia } \\
\pm \text { cough, dyspnea } \pm \text { digestive signs }\end{array}$ \\
\hline III & $\begin{array}{l}\text { Severe single or multiple organ damage Cardiovascular collapse, tachycardia or bradycardia } \pm \\
\text { cardiac rhythm disorders } \pm \text { bronchospasm } \pm \text { digestivesigns } \\
\text { Mucocutaneous signs may be absent or may not appearuntil hemodynamic restoration }\end{array}$ \\
\hline IV & Cardiac arrest \\
\hline
\end{tabular}

Thus, the emergency situations developed for the scenarios cover the following clinical skills:

- Management of an immediate hypersensitivity reaction to iodinated contrast media in CT: Grade II;

- Management of a hypersensitivity reaction to iodinated contrast media in CT: Cardiac arrest (Grade IV);

- The identification of errors associated with care in the context of the room of errors.

Finally, and for the success of this session, the research team proceeded to the realization of trial sessions to test and verify the feasibility and relevance of each clinical case.

\section{Methodology}

\subsection{Data collection}

The study is carried out using a set of valid and reliable measurement tools, as well as quantitative and qualitative data collection methods to enrich the research design.

\subsection{Technical Skills Assessment}

An evaluation of the implementation of the simulation session was conducted to assess the compliance of the students' skills against performance criteria.

Each radiology student was evaluated by a team using a specific evaluation grid for each clinical case, which allowed a score from 0 to 1 to be assigned according to the quality of the procedures performed and the respect of the management algorithms [40]. The total score was calculated by adding the points awarded.

The rubric was based on the OSATS (Objective Structured Assessment of Technical Skills) rubric and was developed by an experienced educational team in accordance with the standards of good practice in the field of radiology [40]. The rubric was pre-tested. 
Similarly, the integration of a standardized patient in the scenarios designed, within the framework of a hybrid simulation, was also a strong point that added realism to the experience lived by the participants.

The Standardized Patient tool is a high-fidelity simulation technique that allows the student to apprehend a patient in all his complexity. Its unique ability to reproduce the emotional and relational dimension makes it a promising technique that offers a safe, full-scale field of experimentation according to the learning objectives.

\subsection{Chamber of errors}

It is a fictitious reconstruction of a scanner room in which a series of 08 errors have been voluntarily implanted according to 4 domains, namely, identity vigilance $N=1$, waste management $\mathrm{N}=1$, hygiene and infectious risks $\mathrm{N}=2$, medication administration errors $\mathrm{N}=4$. The participants will be asked to identify them individually using a survey form for a period of 10 minutes, specifying the title of the error and its location according to 3 choices (caregiver, patient, environment).

\subsection{Learner satisfaction}

At the conclusion of the simulation workshop [41], the student radiology technicians were asked to complete a satisfaction and evaluation questionnaire for the session. This questionnaire consisted of a set of quantitative questions that assessed the students' satisfaction with the simulation workshop using the Likert scale.

In addition, this questionnaire contains open-ended questions:

- The three relevant elements retained through this simulation session for the participants' future professional practices;

- Positive aspects of this simulation from participants' experiences;

- Areas for improvement in the emergency management simulation workshop based on participants' experiences;

- Themes desired by participants for future sessions of the simulation.

\subsection{Ethical consideration}

All participants in this study provided written informed consent before the simulation workshop began. Similarly, the team conducting the simulation sessions provided all necessary clarifications and answered all their questions before the workshop began.

The data processing procedures ensure that no linkage can be made between participants and the data collected during the research. Each participant was assigned a subject file code and no names appear on the data collection instruments. 
Paper-Contribution of Simulation in the Development of the Competences of Future Radiology...

\section{Results}

\subsection{Socio-demographic characteristics of participants}

The sample chosen for our study is made up of $69 \%$ male and $31 \%$ female students, which reflects a dominance of the percentage of males over females.

$(50 \%)$ of the students in this simulation are between 19 and 20 years old, (31.5\%) are 22 years old, (12.5\%) are 21 years old and $(6.25 \%)$ are 30 years old.

$(87.5 \%)$ of the learners are of Moroccan nationality and 2 students are of foreign nationality.

Concerning the educational level of the participating students before coming to ISPITSA, (50\%) have a baccalaureate level, (37.5\%) have a Bac+1, $(6.25 \%)$ have a Bac $+2 /$ DEUG and only one student has already a Bac $+3 /$ licence.

\subsection{Technical skills}

Sixteen students were included in this specific study in "managing emergency and high- risk situations in radiology."

In the first simulation session regarding the management of an immediate hypersensitivity reaction to iodinated contrast media in second-grade $\mathrm{CT}$, the mean score for the session was 06.39/20 (3.25-9).

In addition, the mean score for the second applied simulation session on cardiac arrest management was 06.03/20 (2.25- 8.75).

During the interview, $(100 \%)$ of the students proceeded to verify the identity of the patient, the medical validation of the examination and the consultation of the patient's medical file (biological tests and the examination form).

However, they failed to inform the patient about the procedure for injecting the iodinated contrast medium, the duration of the examination, and the risk of complications.

Regarding risk factors, $(100 \%)$ of the students asked the patient if she had asthma, if she was prone to allergic reactions following the consumption of strawberries, fish or shellfish, or if she had already had an allergic reaction during a previous administration of penicillin. However, no student asked the patient if she had a history of immediate allergic hypersensitivity reaction to a contrast medium and only (43.75\%) of the participants asked her to stop breastfeeding for 48 hours because of the risks to the baby.

In addition, different questions are raised to identify the real factors of failure, pointing the finger at the gap between saying and doing, between concepts and their uses [57].

It was also noted that none of the students prepared or checked for the availability of premedication and emergency medical devices.

Similarly, (100\%) of the students respected the appropriate dosage of PCI to be injected according to the patient's weight and (43.75\%) demonstrated good gestural control adapted to the injection technique.

At the onset of the critical situation, it is noted that $(100 \%)$ of the participants called the radiologist/resuscitator and requested his immediate intervention and only $(25 \%)$ of them stopped the injection of the iodinated contrast product. On the other 
hand, none of them were able to diagnose, characterize, or staging according to the Ring and Messmer classification of the risk situation. Similarly, none of the participants thought to note the time of onset, nor to implement the appropriate protocol for managing the situation.

Finally, none of the participants reported this technique-related adverse event either by filling in the form reserved for this purpose or by reporting it to the head of the department.

\subsection{Learner satisfaction}

All student radiology technicians completed the satisfaction questionnaires.

The analysis of the answers to the questionnaires showed a great satisfaction of the students $(100 \%)$ concerning their participation in this session dedicated to the management of medical imaging emergencies and its adequacy to their expectations.

$(81.3 \%)$ of the participants rated this workshop as a very interesting educational tool and $(18.8 \%)$ who remained rated it as interesting.

Regarding the error room scenario, $8(50 \%)$ of the participants were satisfied with the relevance of the errors implemented during this simulation session and $(37.5 \%)$ very satisfied. A large majority (75\%) of the participants judged simulation in the context of risk management as an effective approach that will allow the improvement of their professional practices in a very satisfactory manner.

Similarly, participants rated their understanding of the risks associated with an ICP injection as very good $(68.8 \%)$ to good $(31.3 \%)$ in the scenarios. $(50 \%)$ of them also mentioned having experienced emotional difficulties during their passage through this experience.

$(62.5 \%)$ of the participants considered this type of simulation to be superior to the teaching they received on the same topic, entitled "what to do when injecting iodinated contrast media and its complications".

Half of the students rated the supervision during the implementation of this simulation session as very satisfactory to satisfactory $(43.8 \%), 31.3 \%$ found the duration of the session to be moderately satisfactory and (68.8\%) found the material used during the simulation to be satisfactory.

$(100 \%)$ of the students appreciated the knowledge acquired during the debriefing, and they believe that the good practice recommendations explained and discussed by the facilitator during this crucial simulation stage are very useful and relevant for their radiology training and for their future practice.

Finally, all the participants think that this simulation complements the practice in training and qualified it as interesting (75\%), enriching (50\%), reassuring (43.8\%) and indispensable $(31.3 \%)$.

Regarding the three relevant elements retained thanks to this simulation session for future radiology technicians, they are: stress management $(100 \%)$, management of a risky situation $(87.5 \%)$, verification of the expiry date $(62.5 \%)$.

The positive aspects to be retained from this simulation workshop according to the participants' experience are: the necessity of learning first aid techniques (100\%), the awareness, during daily practice, of the importance of preventing and anticipating potential risks that threaten the patient $(75 \%)$. 
Other factors may intervene, depending on four factors which directly influence the decision to act towards such an education system: (a) self-confidence, (b) the need to refer to others, (c) the need to " use their experience, and finally, (d) instill the training model [54].

Participants also suggested certain aspects to be improved during the emergency management simulation, namely, having more time to familiarize themselves with the equipment and material available to them (56.25\%) and being exposed to other complex and critical clinical situations to be better equipped to deal with these situations that they experienced as stressful (100\%).

According to Figure 2, the participants recommend for future simulation sessions to address other scenarios and clinical cases, namely "management of a patient with unreported renal failure" (43.75\%), "pediatric management in radiology" (25\%), "management of a polytrauma patient" (18.75\%) and "radiation protection" (15.5\%).

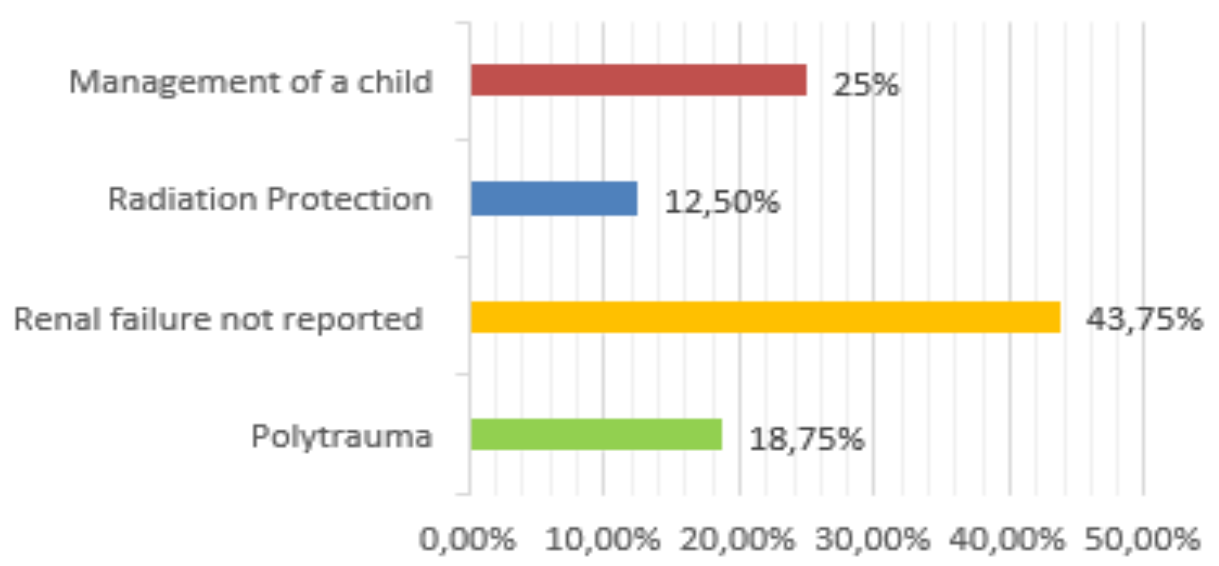

Fig. 2. Participants' desired themes for future simulation sessions.

\subsection{The room of errors}

The exploitation of the results of the error chamber revealed that:

- Only (31.3\%) of the students were able to detect the error of the date of birth on the patient's wristband regarding the area of identity surveillance;

- $(43.8 \%)$ of the students were able to discover that the DASRI needle collector is filled to the maximum with various wastes exceeding its limit regarding the waste management area.

Similarly, for errors concerning the field of hygiene and infectious risk:

- Only 2 participants were able to detect that the patient's sheet is stained with blood;

- No student was able to detect that the bottle of disinfectant has been open for more than 6 months.

Finally, regarding the area of drug administration: 
Paper- Contribution of Simulation in the Development of the Competences of Future Radiology...

- Only 2 students were able to detect the error of the expiration date of the contrast medium to be injected to the patient which was exceeded;

- A large majority of students (93.8\%) were unable to detect that the peripheral venous line dressing is not waterproof and undated;

- No participant could notice that the syringe of the needle for injecting the iodinated contrast medium to the patient is uncapped;

- Only $(18.8 \%)$ of the students were able to detect that the injection dosage of the contrast medium is not mentioned on the patient's chart.

\section{Discussion}

The objectives of this study were to sensitize future radiology technicians to the management of medical imaging emergencies, to evaluate their technical skills when faced with critical situations, and finally to measure their satisfaction with their participation in these simulation sessions focused on the management of radiology emergencies.

This study highlighted the potential threats and risks that a future radiology technician may face during his or her daily practice in a medical imaging department.

The evaluation of the technical skills of the participants in this simulation workshop revealed an insufficient mastery of the management of a hypersensitivity reaction to second grade contrast products and of cardiac arrest (Grade IV).

These results are justified in medical imaging, especially since the probability for a student radiology technician to be confronted with a serious situation during clinical training is low, or even very rare, which makes his clinical experience limited despite the theoretical knowledge he has acquired during his studies.

In addition, the constraints inherent to the internship sites and the "real situation" of taking care of the "real patient" in an emergency situation, where decision making and speed of action play a big role, creates an obligation of efficiency on the part of the care team. Consequently, the student trainee is excluded, and finds it impossible to apply his theoretical learning in gestures and takes the status of an observer.

From this point of view, simulation represents an effective and promising teaching method that allows future health professionals to acquire experience without risk to the patient, and thus to respect the ethical imperative: "Never the first time on the patient". This issue is fundamental and justifies in itself the use of simulation in health care in the context of initial training.

The digital practices of environments depend on certain factors that seem to influence these practices. Indeed, the reflection on the modalities of digital use by students gives rise to several debates [59].

Simulation techniques are widely used in a large number of countries in Europe (England, Sweden, Norway, Germany, Switzerland, Finland, Spain), North America (United States, Canada), or Australia, where they occupy an important place in medical education, in the evaluation of students, in the certification of physicians, in interdisciplinary training, and more generally in programs for improving the quality and safety of care [26]. 
In the context of this study, the research team used simulation in a preventive manner, known as an a priori approach [4], in order to train the ability of future radiology technicians to deal with situations that are potentially or objectively at high risk of error and infrequent, and thus to improve the subsequent management of a patient.

Simulation can also be used in the training of radiology students as an a posteriori risk management method, based on feedback. It consists of using errors and adverse events associated with care (AEHI) to rewrite these situations in simulation in order to identify systemic dysfunctions and decision-making methods [4].

In the daily practice in a medical imaging department, at each step of the management of a patient for radiological examination, risks are possible. They systematically result from the combination of various causes, linked to the direct quality of the care but also to the general organization defects of the material and human environment in which this examination is carried out.

The analysis of the results of this study reveals aspects to be improved concerning certain steps in the examination of the PCI injection, which seem to us to be of primary importance in the management of risks in radiology. Thus, every future radiology technician must welcome the patient, check that the examination has been validated by the radiologist, consult the biological work-up, take a history in search of risk factors that must be taken into account in order to, if necessary, question the PCI injection, and inform the patient of the risks to which he or she may be exposed during the examination $[33,35,37,39]$.

Moreover, the current state of knowledge and the evolution of scientific data in radiology show that the only risk factor identified corresponds to a history of immediate allergic hypersensitivity reaction to a contrast medium [33].

Asthma, use of $\beta$-blockers or food allergy are in no way risk factors for the occurrence of an anaphylactic reaction to a contrast medium [33].

Similarly, immediate allergic reactions to seafood (fish and shellfish), which were considered a risk factor for reaction to ICP on the part of students in this study, were not due to iodine. This history is not a risk factor for allergic reaction to iodinated contrast media [33].

During the examination, the future radiology technician must ensure medical surveillance of the patient during and after the administration of the contrast medium (30 minutes), must be able to identify, characterize, staging (Ring and Messmer classification) and manage intolerance reactions, and must have complete, compliant, frequently and regularly checked management equipment, grouped together in an emergency cart [33, 37, 39].

In this study, evaluation of the management of cardiac arrest demonstrated a lack of proficiency in performing high-quality basic cardiopulmonary resuscitation (CPR). And we recognize that the single most important determinant of survival after cardiac arrest is the presence of a willing and able bystander.

In this regard, simulation training is an essential part of CPR training and there are a multitude of uses for it [21]. Some mannequin studies demonstrate that simulation training, compared with conventional methods, improves performance or produces equivalent results. Numerous educational or temporal biases make it difficult to translate improved performance or survival into real life. A prospective study with resi- 
dents comparing conventional and simulator training showed improved performance in real-life situations [21].

Thus, reducing the risk of a serious undesirable event is based on the involvement of each health professional in order to carry out a health care procedure under the required quality conditions, and on the adoption of a safety culture that allows for the best possible organization of care, in terms of preparation, execution, coordination, verification and communication [4, 21, 39].

Among the methodological tools of health simulation and risk management that are part of a global approach to quality of care, we find the error room, which is part of an a posteriori approach to raise awareness of the pedagogy of error and promote playful learning in a real context [4]. Thus, several pedagogical approaches can reinforce the play-based learning approach, especially at a distance, which thus represents a powerful vector for promoting socio-professional skills [52].

The error room is a health simulation tool based on a learning-by-doing approach. It is a very common tool in English-speaking countries. Several hospitals regularly use it for medical and nursing students as well as for physician assistants when they start their career. Initial positive experiences have also been gathered in Switzerland. In Canada, the idea has been taken up in a pediatric hospital (under the title "crib of horrors"), where this tool has been used by multiprofessional teams [41, 42, 43].

Thus, in order to develop and germinate this positive culture of risk, the research team has set up an "error room". This involves the deliberate introduction of errors into a simulated care or management environment. These errors are diversified, depending on the educational objectives. This method, which is the frontier of simulation, encourages future professionals to detect and report errors and to change their view of errors, which then becomes a factor for improvement [4, 43, 44, 45].

According to Ford, the rate of errors in critical care can be reduced by a simulation-based training program compared to a traditional didactic program. In his observational study of a total of 880 injections on 76 patients performed by 24 nurses, Ford found a significant reduction in the error rate 4 weeks after training (from $30.8 \%$ to $4 \%$ ) but also 8 to 12 weeks later $(6.2 \%)[43,46]$.

Similarly, various studies in radiological simulation have demonstrated better knowledge transfer (improved performance and diagnostic learning scores) in errorbased learning scenarios, as opposed to error avoidance methods [47, 48]. The search for errors during a simulation session, as well as reflection on their management and potential consequences, reinforces skill acquisition [48].

In addition, a study aimed to identify the obstacles hindering the use of the CAEx "Computer-Assisted Experimentation" in science. Indeed, the results showed that the integration of the CAEx comes up against several obstacles despite the efforts made by the Ministry of Education and Vocational Training [55].

Also, published work [43] shows the effectiveness of errors in learning and the impact of the voluntary use of errors to improve error analysis and ensure sustainable teaching.

Finally, the evaluation of the simulation workshop applied to the management of medical imaging emergencies revealed a high level of satisfaction among the participants following this experience, which they described as interesting, enriching, reassuring and 
superior to the teaching they received as part of their training. They suggest combining this relevant method with internships for optimal learning and better performance.

In this respect, the effectiveness of simulation in health care is no longer in question. It has been shown to be effective in acquiring technical skills [27], particularly when combined with clinical practice, in comparison with theoretical teaching associated with internships [27].

Similarly, there is a large body of medical literature demonstrating not only the seamless integration of medical simulation into educational programs, but also the positive impact of these techniques in the ultimate goal of improving teaching and training [21].

From all these elements collected through this study, it is appropriate to suggest the integration and inclusion of simulation, as an innovative approach, in the training curriculum of radiology technicians, thus allowing trainees to become aware of the notion of risk, to become aware of the management of risks associated with medical imaging care and to relate it to real-life experience, thus better anchoring and consolidating theoretical knowledge.

\section{Conclusion}

This study highlighted the importance of integrating simulation applied to medical imaging emergency management into the training curriculum of future radiology technicians at ISPITSA.

Indeed, choosing as a pedagogical approach the teaching by the various techniques of simulation, in initial training, will allow an improvement of the competences of the students necessary for an optimal performance in particular in complex, high risk, unpredictable environments of care and whose prognosis is vital.

Thus, putting forward a reflective approach is a crucial step to evolve the future of radiology technician training and to impulse a new dynamic in the training of tomorrow's professionals.

\section{Declaration of interest}

The authors declare that they have no conflicts of interest in relation to this article.

\section{$7 \quad$ References}

[1] Langen HJ,. (1996). Training program for radiologic technologists for performing chest Xrays at inspiration in uncooperative children. RöFo - Fortschritte auf dem Gebiet der Röntgenstrahlen und der bildgebenden Verfahren,. https://doi.org/10.1055/s-00000066

[2] Wright Dl, Rolland JP, Kancherla AR. (1995). Using virtual reality to teach radiographic positioning. Radiologic technology.

[3] Matsubara K,. (2011). A head phantom study for intraocular dose evaluation of 64-slice multidetector CT examination in patients with suspected cranial trauma. Eur JRadiol,. https://doi.org/10.1016/j.ejrad.2010.02.002 
Paper- Contribution of Simulation in the Development of the Competences of Future Radiology...

[4] Pelaccia T. (2016). Comment mieux former et évaluer les étudiants en médecine et en sciences de la santé ? De Boeck Supérieur. https://doi.org/10.1051/pmed/2017028

[5] Barrows HS. (1987). Simulated (standardized) patients and other human simulations. Chapel Hill: Health Sciences Consortium.

[6] Nestel D,. (2011). The use of simulated patients in medical education: Guide Supplement 42.1 - Viewpoint. Medical teacher.

[7] Kneebone R,. (2006). The human face of simulation: patient-focused simulation training. Academic Medicine.

[8] Normes des meilleures pratiques de l'INACSL : Simulation Conception de la simulation, Comité de normalisation de l'International Nursing Association for Clinical Simulation and Learning. Elsevier Inc, 2016.

[9] Oriot D, Alinier G. (2019). La simulation en santé, le débriefing clés en mains. Elsevier Masson.

[10] Fiche de recommandation pour la pratique clinique " Produits de contraste et Allergie : Hypersensibilité de type immédiat" SFR- CIRTACI- octobre 2009. https://doi.org/10. 1016/s0221-0363(09)74755-9

[11] GUIDE des technologies de l'imagerie médicale et de la radiothérapie de Jean-Philippe Dillenseger, Elisabeth Moerschel, Claudine Zorn, 2e édition. Octobre 2016.

[12] Société Française de Radiologie (SFR) et Comité Interdisciplinaire de Recherche et de Travail sur les Agents de Contraste en Imagerie (cirtaci). Recommandations pour la pratique Clinique. Produits de contraste et allergie : Hypersensibilité de type immédiat. Octobre 2009. https://doi.org/10.1016/s0221-0363(07)81004-3

[13] Le Collège National de Pharmacologie Médicale (CNPM). Produits de contraste et médicaments radio-pharmaceutiques. Juillet 2018.

[14] Fiches pratiques d'utilisation des produits de contraste -cirtaci- Février 2020.

[15] Ring J, Messmer K. (1977). Incidence and severity of anaphylactoid reactions to colloid volume substitutes. Lancet. https://doi.org/10.1016/s0140-6736(77)91953-5

[16] Elisabeth Schouman-claeys. (2012). Produits De Contraste Et Gestion Des Risques: Aménager Des Règles De Conduite Simples. Health Management, Volume 5 - Numéro 1.

[17] Société Française de Médecine d'Urgence (sfmu). (2012). Les réactions allergiques. Les bonnes pratiques. Urgences.

[18] Chantal Zimmermann; Prof. Dr. David Schwappach. (2019). Fondation pour la Sécurité des patients Suisse. Apprentissage interactif grâce à une chambre des erreurs : Guide d'utilisation. Sécurité des patients Suisse.

[19] Farnan JM, Gaffney S, Poston JT, Slawinski K, Cappaert M, Kamin B,. (2016). Patient safety room of horrors: A novel method to assess medical students and entering residents' ability to identify hazards of hospitalisation. BMJ Qual Saf. https://doi.org/10.1136/bmjqs2015-004621

[20] Trouiller P., Benhamou D. (2017). Chambre des erreurs. MAPAR.

[21] Daupin J, Atkinson S, Bédard P, Pelchat V, Lebel D, Bussières JF. (2016). Medication errors room: a simulation to assess the medical, nursing and pharmacy staffs' ability to identify errors related to the medication-use system. J Eval Clin Pract. https://doi.org/10 $.1111 /$ jep. 12558

[22] Joret-Descout P, Te Bonle F, Demange C, Bechet M, Da Costa M, Camus G, Bohand X. A. (2015). training medication errors room: simulate to better train health professionals. J Pharm Belg. https://doi.org/10.1016/j.phclin.2014.10.047

[23] Daniel G. Ford, Amy L. Seybert, Pamela L. Smithburger, Lawrence R. Kobulinsky, Joseph T. Samosky, Sandra L. Kane-Gill. (2010) Impact of simulation-based learning on medication error rates in critically ill patients. Intensive Care Med. https://doi.org/10.1007 $\underline{\text { s } 00134-010-1860-2}$ 
Paper- Contribution of Simulation in the Development of the Competences of Future Radiology...

[24] Dyre L, Tabor A, Ringsted C, Tolsgaard MG. (2017). Imperfect practice makes perfect: error management train of learning.Med Educ. https://doi.org/10.1111/medu.13208

[25] Gardner A, Rich M. (2014). Error management training and simulation education. Clin Teach.

[26] Daaif J., Zerraf S., Tridane M., Benmokhtar S., Belaaouad S. (2019). Technological Innovation in Teaching and Research in Chemical Science: Development of a Computer Application for the Simulation of the Practical Works of Crystallography. International Journal of Recent Technology and Engineering (IJRTE) ISSN: 2277-3878, Volume-8 Issue-3. https://doi.org/10.35940/ijrte.c4665.098319

[27] Daaif J., Zerraf S., Tridane M., Benmokhtar S., Belaaouad S. (2019). Pedagogical engineering to the teaching of the practical experiments of chemistry: Development of an application of three dimensional digital modelling of crystalline structures. Cogent Education, 2019, 6(1), 1708651. https://doi.org/10.1080/2331186x.2019.1708651

[28] Muftisada, J., Bassiri, M., Tridane, M., Belaaouad, S. (2019). Engineering Emotional Intelligence in Moroccan University. International Journal of Advanced Trends in Computer Science and Engineering, Volume 8 No. 1.4, https://doi.org/10.30534/ijatcse/2019/ $\underline{4481.42019}$

[29] Bassiri, M., Boulahouajeb, A., Aichi, Y., Belaaouad, S., Radid, M. (2018). Distance learning - A powerful vector of the enhancement of socio-professional competences: Case of the training of contract teachers in Regional Centre of Training and Education, Morocco. ASIA LIFE SCIENCES Supplement 16(1): 11-19. The Asian International Journal of Life Sciences.

[30] Muftisada, J., Daaif, J., Tridane, M., Belaaouad, S. (2020). Use of smartphones in learning and techno-pedagogical integration of artificial intelligence tools as a prospect for intelligent learning: Case of moroccan students from hassan ii university of Casablanca International Journal of Emerging Trends in Engineering Research, 2020, 8(9), pp. 6496-6504, 252. https://doi.org/10.30534/ijeter/2020/252892020

[31] Habybellah Z., Bassiri, M., Belaaouad S., Radid, M., Benmokhtar, S. (2019). Training and Development of Professional Skills: An Analysis of activity in Professional Skills. International Journal of Advanced Trends in Computer Science and Engineering. 8. 2029-2033. https://doi.org/10.30534/ijatcse/2019/28852019

[32] Ouargui, Y., Gourja, B., Tridane, M., Belaaouad, S. (2019). Use of Computer-Assisted Experimentation (CAEx) in Teaching Science in Moroccan High Schools. International Journal of Web Applications. 11. 119. https://doi.org/10.6025/ijwa/2019/11/4/119-124

[33] Aichi Y., BASSIRI, M., BENMOKHTAR, S. BELAAOUAD, S. (2020). Paper-EAssessment as a Vector for Identifying and Increasing the Validation of Advice Within EAssessment as a Vector for Identifying and Increasing the Validation of Advice Within Professional Organizations. International Journal of Interactive Mobile Technologies (iJIM). Vol. 14, No. 20, 2020. 107-116. https://doi.org/10.3991/ijim.v14i20.15663

[34] Maziane, M., Bassiri, M., Benmokhtar, S., Belaaouad, S. (2020). Engineering analysis of teaching practices and learning strategies guided by the principles of Cognitive Psychology and Information technology. International Journal of Advanced Trends in Computer Science and Engineering. Volume 9. 212 - 217.

[35] Chiboub, N., Tridane, M., Belaaouad, S. (2019). The Importance of Teacher Cooperation in the Process of School Guidance for High School. International Journal of Innovative Technology and Exploring Engineering (IJTTEE). ISSN: 2278-3075, Volume-9 Issue-1, pp (3747-3751). https://doi.org/10.35940/ijitee.a4810.119119

[36] Fikri, R., Belhabra, M., Khouya, E., Tridane, M., Belaaouad, S. (2018). The use of digital modalities among Moroccan PhD students: Case of Hassan II University of Casablanca. 
Paper- Contribution of Simulation in the Development of the Competences of Future Radiology...

ASIA LIFE SCIENCES Supplement 16(1): 239-245. The Asian International Journal of Life Sciences.

\section{Authors}

Asma Fadi is a Permanent teacher at the Higher Institute of Nursing Professions and Health Techniques of Agadir-Morocco since 2019. Director of the Higher Institute of Nursing Professions and Health Techniques of Agadir from June 2015 until June 2019. PhD student researcher, Engineering of Training and Didactics of Sciences and Techniques. Master in Sanitary Administration and Public Health from the National School of Public Health Rabat- Morocco. Master of Paramedical Studies Section: Paramedical Education, Health Career Training Institute Rabat- Morocco, cohort: 2002-2004 (asmafadi2017@gmail.com).

Fatima Zahra Ramdani is a Radiology Technologist at the regional hospital center of Guelmim, Morocco. PhD student researcher in laboratory in health sciences, National School of Applied Sciences of Agadir-Morocco. Master in Nursing and Health Technology Pedagogy, Higher Institute of Nursing Professions and Health Techniques Agadir-Morocco (2017-2019).

Khadija Legrouri is a Principal Professor, Higher Institute of Nursing Professions and Technics Health, ISPITS Agadir, Morocco, June 2013 to date. Quality Insurance Manager, Ministry of Health - Morocco, June 2002 to June 2013. Educational Coordinator of the Master's program in Nursing Sciences and Health Techniques. Educational Coordinator of the Rehabilitation and Rehabilitation.

Jabran Daaif is a $\mathrm{PhD}$ student researcher in engineering training and didactics of sciences and techniques. He had his Masters in Software Quality. He works on the engineering of techno-educational tools in the service of physical and chemical sciences and the development of simulation and modeling systems.

El Hassane Khouya is a Principal Professor, Centre Régional des Métiers de l'Education et de la Formation - Souss Massa, Mai 2011 to date. Deputy Director, Responsible for the Qualification Cycle. Doctor of Philosophy Chemistry and Education Chemistry, 2002, Hassan II University, Casablanca-Morocco. Coordinator of « Learning System and Specialized Didactics » Team. Co-Founder of the « Interdisciplinary Research Laboratory in Education, Training, Engineering and Modeling ».

Said Benmokhtar is a Research professor at Hassan II University of Casablanca, Ben M'Sik Faculty of Sciences. Very strong skills in X -ray Diffraction, Crystal Chemistry, Crystallography on powder.

Said Belaaouad is a Research professor at the Faculty of Science Ben M'sik Hassan University II. Director of the Laboratory of Physical Chemistry of Materials. He works with materials based on phosphates, phosphites and metal oxides (spinels, perovskites) for various applications: fertilizers, corrosion inhibitors, frequency doublers in ONL nonlinear optics, renewable energies, the generation and storage of water. chemical and electrical energy in batteries and cells.

Article submitted 2021-05-06. Resubmitted 2021-06-07. Final acceptance 2021-06-08. Final version published as submitted by the authors. 